\title{
Profile of vortioxetine in the treatment of major depressive disorder: an overview of the primary and secondary literature
}

This article was published in the following Dove Press journal:

Therapeutics and Clinical Risk Management

12 August 2015

Number of times this article has been viewed

\section{Marc Kelliny \\ Paul E Croarkin \\ Katherine M Moore \\ William $\vee$ Bobo}

Department of Psychiatry and Psychology, Mayo Clinic, Rochester, MN, USA
Correspondence: William V Bobo Department of Psychiatry and Psychology, Mayo Clinic, 200 First Street SW, Generose 2A, Rochester, MN 55905, USA

$\mathrm{Tel}+\mid 5072559412$

Email bobo.william@mayo.edu
Abstract: This article reviews the pharmacological profile and available efficacy and tolerability/ safety data for vortioxetine, one of the most recent antidepressant drugs to be approved in the USA for the treatment of major depressive disorder (MDD) in adults. The efficacy of vortioxetine for treating MDD in adults is supported by eight positive short-term (6- to 12-weeks) randomized, placebo-controlled trials, and one positive randomized, double-blind, 52-week relapse prevention trial. Based on pooled data from short-term randomized trials and from longer-term studies, vortioxetine appears to be well tolerated and to have a low incidence of adverse effects on sexual functioning. Vortioxetine also appears to be effective for treating symptoms of MDD in the elderly based on the results of one randomized trial for which recruitment was focused on this specific population. Nevertheless, effectiveness studies that directly compare the clinical effects of vortioxetine with other established antidepressant drugs are lacking, and there is no evidence as yet that vortioxetine is more clinically effective than other types of antidepressants. Some preliminary suggestions concerning the place of vortioxetine among the broad range of pharmacological treatments for adults with MDD are provided.

Keywords: vortioxetine, Lu AA21004, pharmacological profile, pharmacokinetics, drug interactions, adverse effects/side effects/safety, depression, major depression

\section{Introduction}

Major depressive disorder (MDD) is one of the most prevalent illnesses in medicine, affecting over 350 million people worldwide. ${ }^{1}$ MDD is characterized by pronounced changes in mood (persisting depression or anhedonia) coupled with distinct psychological and vegetative changes, including sleep and/or appetite disturbance, subjective fatigue, loss of motivation and drive, ruminative feelings of guilt and despair, problems maintaining mental focus, and suicidal thinking and behavior. ${ }^{2}$ The adverse effects of MDD on overall health are equivalent to those imposed by other serious chronic diseases or health conditions such as cardiac disease, diabetes, arthritis, and asthma. ${ }^{3}$ MDD is also costly, with pooled estimates of global disease burden indicating that MDD was the second leading cause of years of life lived with a disability in 2010, accounting for $8.2 \%$ of global years of life lived with a disability. ${ }^{4}$ By 2020, MDD is predicted to become the leading cause of disability worldwide. $^{5}$

For many patients with MDD, the illness course is episodic, while others may experience more protracted episodes that can last for several years. ${ }^{6-8}$ For moderate to severe cases, pharmacotherapy with antidepressants is considered to be the first line of treatment, preferably in conjunction with evidence-supported psychotherapy., 90 
A large number of antidepressants are available for the treatment of MDD; however, numerous unmet needs in MDD treatment remain. A meta-analysis of published randomized trials of antidepressants for MDD reported a positive antidepressive response rate of $53 \%$, a rate of response that was significantly higher than the placebo response rate of $36 \%,{ }^{11}$ but numerically lower than the anticipated response rate of $60 \%-70 \%$ reported in earlier reviews of controlled studies. ${ }^{12}$ Rates of acute and sustained symptom remission, which is the desired outcome of antidepressant treatment, ${ }^{13}$ are even lower than for a positive antidepressive response. ${ }^{14-16}$

To manage these challenges, practitioners have applied numerous strategies for increasing antidepressive response to pharmacotherapy, including combining drug treatment with evidence-supported psychotherapies, combining antidepressants of differing pharmacological classes, and combining antidepressants with non-antidepressant psychotropic medications. ${ }^{17-20}$ While these strategies are helpful for many, a substantial number of MDD patients who receive antidepressant treatment still fail to achieve clinically meaningful improvement. Safe and more effective pharmacotherapies for patients with MDD are urgently needed.

In September 2013, the US Food and Drug Administration approved vortioxetine (under the brand name Brintellix ${ }^{\circledR}$, formerly designated as Lu AA21004) for the treatment of MDD in adults. This non-systematic overview summarizes the pharmacological profile of vortioxetine, examines the available clinical trial data supporting its use for treating adults with MDD, and discusses its role in the context of other available antidepressant drugs.

\section{Data sources}

We conducted a MEDLINE database literature search in February 2015 using the key search terms, vortioxetine and Lu AA21004, combined with additional terms that included pharmacological profile, pharmacokinetics, drug interactions, adverse effects/side effects/safety, depression, major depression, and major depressive disorder. We identified relevant systematic reviews, meta-analyses, randomized trials, and important non-randomized studies. The reference sections of retrieved papers were reviewed in order to locate additional reports that were not identified in the initial MEDLINE search. Finally, we augmented the MEDLINE search with information on additional randomized trials of vortioxetine for MDD in adults as posted on www.ClinicalTrials.gov.

\section{Pharmacological profile Chemistry}

The molecular weight and molecular formula for vortioxetine are $298.45 \mathrm{~g} / \mathrm{mol}$ and $\mathrm{C}_{18} \mathrm{H}_{22} \mathrm{~N}_{2} \mathrm{~S}$, respectively. ${ }^{21}$ Vortioxetine is classified under the International Union of Pure and Applied Chemistry name 1-[2-(2,4-dimethylphenyl) sulfanylphenyl]piperazine. ${ }^{21}$ The biochemical and pharmacological properties of vortioxetine are further summarized in Table 1.

\section{Pharmacodynamics}

Although the pathophysiology of MDD is not precisely understood, a large body of evidence indicates that disruptions in serotonergic, noradrenergic, and dopaminergic neurotransmission are important factors, and that intact serotonergic neurotransmitter systems are needed to maintain a positive clinical response. ${ }^{22-24}$ Vortioxetine binds with high affinity to (and potently inhibits the activity of) the serotonin transporter (SERT, $\mathrm{K}_{\mathrm{i}} 1.6 \mathrm{nM}$ ). ${ }^{25,26}$ This property is believed to be the principal mechanism underlying the antidepressive effect of vortioxetine and numerous other antidepressants. $^{27}$

In addition to SERT blockade, in vitro neuroreceptor binding studies using human and rodent cell lines have shown that vortioxetine interacts with numerous serotonin receptors. ${ }^{28}$ Vortioxetine is a potent antagonist at serotonin $5-\mathrm{HT}_{3}$ receptors $\left(\mathrm{K}_{\mathrm{i}} 3.7 \mathrm{nM}\right) ;{ }^{25}$ a weaker antagonist at $5-\mathrm{HT}_{7}$ $\left(\mathrm{K}_{\mathrm{i}} 19 \mathrm{nM}\right)$ and $5-\mathrm{HT}_{1 \mathrm{D}}$ receptors $\left(\mathrm{K}_{\mathrm{i}} 54 \mathrm{nM}\right)$; a partial agonist at $5-\mathrm{HT}_{1 \mathrm{~B}}$ receptors $\left(\mathrm{K}_{\mathrm{i}} 33 \mathrm{nM}\right)$; and a full agonist at 5- $\mathrm{HT}_{1 \mathrm{~A}}$ receptors $\left(\mathrm{K}_{\mathrm{i}} 15 \mathrm{nM}\right){ }^{25,28,29}$

The clinical significance of the binding activity of vortioxetine at these serotonin receptors is unknown. However, a number of potentially useful clinical effects have been ascribed to some of them. ${ }^{27,30-32}$ For example, in preclinical studies, administration of a $5-\mathrm{HT}_{3}$ antagonist, ${ }^{29} 5-\mathrm{HT}_{1 \mathrm{D}}$ antagonist, ${ }^{33}$ or $5-\mathrm{HT}_{7}$ antagonist ${ }^{34}$ in the presence of SERT blockade (typically with a selective serotonin reuptake inhibitor [SSRI]) have all been shown to facilitate enhanced neuronal serotonin release, as compared with an SSRI alone. Vortioxetine administration has been shown to induce higher levels of extracellular serotonin release with subchronic (3 days) dosing than SSRI administration in the rodent hippocampus and medial prefrontal cortex despite similar SERT occupancies. ${ }^{35}$ This effect is hypothesized to be related to the binding activities of vortioxetine at serotonin receptors, coupled with SERT blockade. ${ }^{28}$ 
Table I Clinical summary of vortioxetine $\left(\right.$ Brintellix $\left.{ }^{\circledR}\right)$

\begin{tabular}{|c|c|}
\hline Class of agent & $\begin{array}{l}\text { - N06AX26, other, antidepressants (ATC) }{ }^{\mathrm{a}} \\
\text { - Antidepressant with multimodal mechanism of action }{ }^{\mathrm{b}}\end{array}$ \\
\hline Formulation & Solid oral tablets $(5,10$, and $20 \mathrm{mg})$ \\
\hline Starting dose & $10 \mathrm{mg} /$ day, once daily (then increase as tolerated to target dose of $20 \mathrm{mg} /$ day) \\
\hline Effective dose range & 5 to $20 \mathrm{mg}$, once daily \\
\hline Approved indication & Major depressive disorder, adults \\
\hline Pharmacokinetics & $\begin{array}{l}\text { - Oral bioavailability: } 75 \% \\
\text { - } \mathrm{T}_{1 / 2}: 57-66 \text { hours } \\
\text { - Metabolism: CYP2D5 (primary), CYP3A4/5, CYP2C9, CYP2C19, CYP2C8, CYP2A6, and CYP2B6 }\end{array}$ \\
\hline Pharmacodynamics & $\begin{array}{l}\text { - SERT inhibitor } \\
\text { - Antagonist: } 5-\mathrm{HT}_{3}, 5-\mathrm{HT}_{7}, 5-\mathrm{HT}_{\mathrm{ID}} \\
\text { - Partial agonist: } 5-\mathrm{HT}_{\mathrm{IB}} \\
\text { - Agonist: } 5-\mathrm{HT}_{\mathrm{IA}}\end{array}$ \\
\hline $\begin{array}{l}\text { Drug interactions/ } \\
\text { dose adjustments }\end{array}$ & $\begin{array}{l}\text { - Reduce dose by 50\% when administered to a pharmacogenetic poor CYP2D6 metabolizer, or when given } \\
\text { concomitantly with a strong CYP2D6 inhibitor. } \\
\text { - Higher doses may be required when given concomitantly with a strong CYP3A4 inducer. } \\
\text { - No dose adjustments are required on the basis of patient age, sex, or race. } \\
\text { - No dose adjustments are required in patients with mild to moderate renal or hepatic impairment. }\end{array}$ \\
\hline Contraindications/precautions & $\begin{array}{l}\text { - Coadministration with a monoamine oxidase inhibitor is contraindicated. } \\
\text { - Use in patients with severe hepatic impairment is not recommended. } \\
\text { - The vortioxetine drug label has the antidepressant class warning about the potential for increased risk of } \\
\text { suicidal thoughts and behavior in children, adolescents, and young adults. }{ }^{.}\end{array}$ \\
\hline Pregnancy and breastfeeding & $\begin{array}{l}\text { - US Food and Drug Administration safety in pregnancy category: } \mathrm{C}^{d} \\
\text { - Lactational safety profile of vortioxetine: unknown }\end{array}$ \\
\hline Use in children and adolescent & - The effectiveness and safety of vortioxetine for any indication has not been studied in children or adolescents \\
\hline Geriatric adults & $\begin{array}{l}\text { - Positive randomized trial in geriatric adults (Table 3) } \\
\text { - No dose adjustment required based on age }\end{array}$ \\
\hline
\end{tabular}

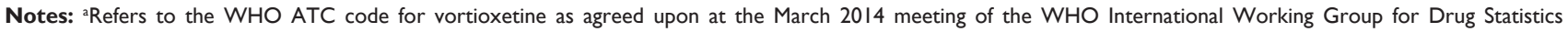
Methodology (see http://www.who.int/medicines/publications/druginformation/issues/Druglnformation20I4_Vol28-4/en/). 'Refers to vortioxetine's designation under the psychotropic medication reclassification system proposed by the European College of Neuropsychopharmacology, in collaboration with the American College of Neuropsychopharmacology, the Asian College of Neuropsychopharmacology, the International College of Neuroopsychopharmacology, and the International Union of Basic and Clinical Pharmacology (see http://www.ecnp.eu/projects-initiatives/nomenclature.aspx). ${ }^{c}$ Close monitoring for the emergence of suicidal thoughts and behavior is recommended for patients at any age who are started on antidepressant treatment. Vortioxetine has not been studied for any indication in children and adolescents. ${ }^{d}$ No adequately controlled studies of vortioxetine in pregnant women. The package label references no teratogenic effects in rodents administered $58-77$ times the maximum recommended human dose $(20 \mathrm{mg})$ during pregnancy. Vortioxetine should be used in pregnancy only if potential benefits outweigh potential risks to the developing fetus. Abbreviations: ATC, Anatomic Therapeutic Classification; WHO, World Health Organization; $\mathrm{T}_{12}$, mean elimination half-life; CYP, cytochrome P450; SERT, serotonin transporter.

Serotonergic 5 - $\mathrm{HT}_{1 \mathrm{~A}}$ receptors are more established treatment targets for both MDD and for anxiety disorders, which often occur comorbidly in patients with MDD. ${ }^{36-38}$ Indeed, several 5- $\mathrm{HT}_{1 \mathrm{~A}}$ partial agonists have been shown to improve depressive symptoms, either alone or as pharmacological adjuncts to SSRIs, ${ }^{19,39}$ although not all studies are in agreement. ${ }^{39}$ Additionally, pindolol, a non-selective $\beta$-adrenergic receptor antagonist and 5- $\mathrm{HT}_{1 \mathrm{~A} / \mathrm{B}}$ partial agonist, has been shown to have a modest effect in terms of enhancing the early (at 2 weeks) and later (at 6 weeks) positive antidepressive response with SSRIs in patients with non-treatmentresistant $\mathrm{MDD},{ }^{40}$ although several studies have not replicated these effects. ${ }^{41-44}$ In the case of vortioxetine, it remains unclear whether its full (rather than partial) agonist effect at 5-HT ${ }_{1 \mathrm{~A}}$ receptors translates into clear clinical advantages over other agents with regard to shortening the time to positive treatment response or enhancing short-term or longer-term efficacy.
Vortioxetine does not appear to interact significantly with the norepinephrine transporter or dopamine transporter. Still, vortioxetine administration has been shown to increase extracellular levels of norepinephrine, dopamine, and nonmonoamine neurotransmitters including acetylcholine and histamine. ${ }^{28,29}$ These effects are also thought to be related to the interaction between vortioxetine and various serotonin receptors. ${ }^{28}$

\section{Pharmacokinetics and metabolism Absorption and distribution}

Vortioxetine is readily absorbed following oral ingestion, with post-administration peak plasma concentrations achieved within 7-11 hours, and an oral bioavailability of $\sim 75 \%$ that is unaffected by food. ${ }^{45}$ The drug is highly protein-bound ( $98 \%$ ), and has a large volume of distribution $(2,600 \mathrm{~L}){ }^{46}$ 


\section{Metabolism and elimination}

Vortioxetine has a mean elimination half-life of 57-66 hours. ${ }^{46}$ Vortioxetine undergoes extensive hepatic metabolism, mainly through oxidation via the cytochrome P450 (CYP)2D6 isoenzyme. Numerous other isoenzymes contribute to the metabolism of vortioxetine, including CYP3A4/5, CYP2C9, CYP2C19, CYP2C8, CYP2A6, and CYP2B6. ${ }^{47}$ None of the metabolites of vortioxetine are thought to be pharmacologically active. ${ }^{48}$ Pharmacogenetic poor metabolizers at CYP2D6 have been shown to have approximately twice the vortioxetine plasma concentration as extensive (normal) metabolizers; ${ }^{48}$ therefore, the manufacturer has recommended a maximum oral daily vortioxetine dose of $10 \mathrm{mg}$ for this population. ${ }^{45}$

\section{Modifying factors}

The clearance of vortioxetine does not appear to be affected by age, race, or sex. ${ }^{45}$ Additionally, neither mild to moderate hepatic nor renal impairment appears to impact the clearance of vortioxetine..$^{45}$ Therefore, dose adjustments based on demographic characteristics and mild to moderate hepatic or renal impairment are not required. The safety of vortioxetine has not been evaluated in patients with severe hepatic impairment. As such, the manufacturer recommends avoiding vortioxetine in that population. ${ }^{45}$

\section{Drug interactions}

Coadministration of strong CYP2D6 inhibitors has been shown to increase serum concentrations of vortioxetine, while strong CYP2D6 inducers have been shown to have the opposite effect. ${ }^{45,47,48}$ Therefore, the manufacturer has recommended lowering the total daily dose of vortioxetine by $50 \%$ when using it concomitantly with strong CYP2D6 inhibitors (eg, fluoxetine, paroxetine, bupropion, and quinidine), and considering an increase in vortioxetine dose when coadministered with strong CYP450 inducers (eg, rifampicin, carbamazepine, and phenytoin). ${ }^{45,46}$ Like other antidepressants, vortioxetine is contraindicated for patients taking monoamine oxidase inhibitor antidepressants. ${ }^{45}$

\section{Clinical efficacy}

\section{Individual studies for acute treatment of major depression}

All short-term, acute-phase studies of vortioxetine for treating adults with MDD used fixed-dose, parallel-group designs, and enrolled patients with no evidence of treatmentresistant depression (generally defined as having failed to respond to two adequate antidepressant treatments of at least
6 weeks' duration). ${ }^{49-59}$ Table 2 highlights the considerable variability between studies with respect to type of study participants, treatment settings, minimum severity of depressive symptoms for inclusion in the study (MontgomeryÅsberg Depression Rating Scale [MADRS] total scores 22-30), vortioxetine doses (2.5-20 mg daily), and length of follow-up (6-12 weeks). One published positive study compared the clinical effects of flexibly dosed vortioxetine and agomelatine in patients who did not respond adequately to SSRIs or serotonin-norepinephrine reuptake inhibitors (SNRIs).$^{56}$ One study focused exclusively on elderly adults (aged $\geq 65$ years). ${ }^{51}$ Only eleven of the 14 reviewed studies were published at the time of review. The risk of bias was assessed as being low or unclear across the nine published studies as determined using the Cochrane Risk of Bias Assessment tool (Figure 1). Three studies were assessed as having low bias risk in all domains. ${ }^{49,51,56}$ Although the bias risk assessment was generally low across studies for randomization sequence generation, allocation concealment, and selective outcome reporting, the presence of bias in other domains (including masking of clinical raters) cannot be excluded. There was insufficient methodological detail for the unpublished studies, as posted on www.ClinicalTrials.gov, to permit a thorough risk of bias assessment.

Of the 14 reviewed short-term randomized trials, eight were positive (one study unpublished), ${ }^{49-51,54-56,58}$ five were negative (two studies unpublished), ${ }^{52,53,59}$ and one was considered a failed study (Table 3). ${ }^{57}$ The eight positive studies had generally high completion rates and demonstrated statistically significant improvements in depressive symptoms (as measured by the MADRS ${ }^{60}$ or 24-item version of the Hamilton Depression Rating Scale [HDRS] $]^{61}$ ), relative to placebo, at vortioxetine doses ranging from $5 \mathrm{mg}$ to $20 \mathrm{mg}$ daily. Rates of positive response with vortioxetine in the positive studies ranged widely, from as low as $34 \%$ to as high as $78 \%$ (versus $28 \%-45 \%$ with placebo). Vortioxetine-associated remission rates also varied substantially between studies, from as low as $21 \%$ to as high as $55 \%$ (versus $17 \%-27 \%$ with placebo).

A number of positive studies (one unpublished) included an active control group that received fixed doses of venlafaxine $(225 \mathrm{mg})$ or duloxetine $(60 \mathrm{mg}))^{49,51,54}$ However, the individual trials were not statistically powered to permit direct comparisons of antidepressive effects between vortioxetine and either venlafaxine or duloxetine. Only one randomized trial directly compared the antidepressive effects of vortioxetine (10-20 mg) and another antidepressant (agomelatine, $25-50 \mathrm{mg}){ }^{56} \mathrm{In}$ that study, vortioxetine was found to be non-inferior to agomelatine (at a non-inferiority margin 


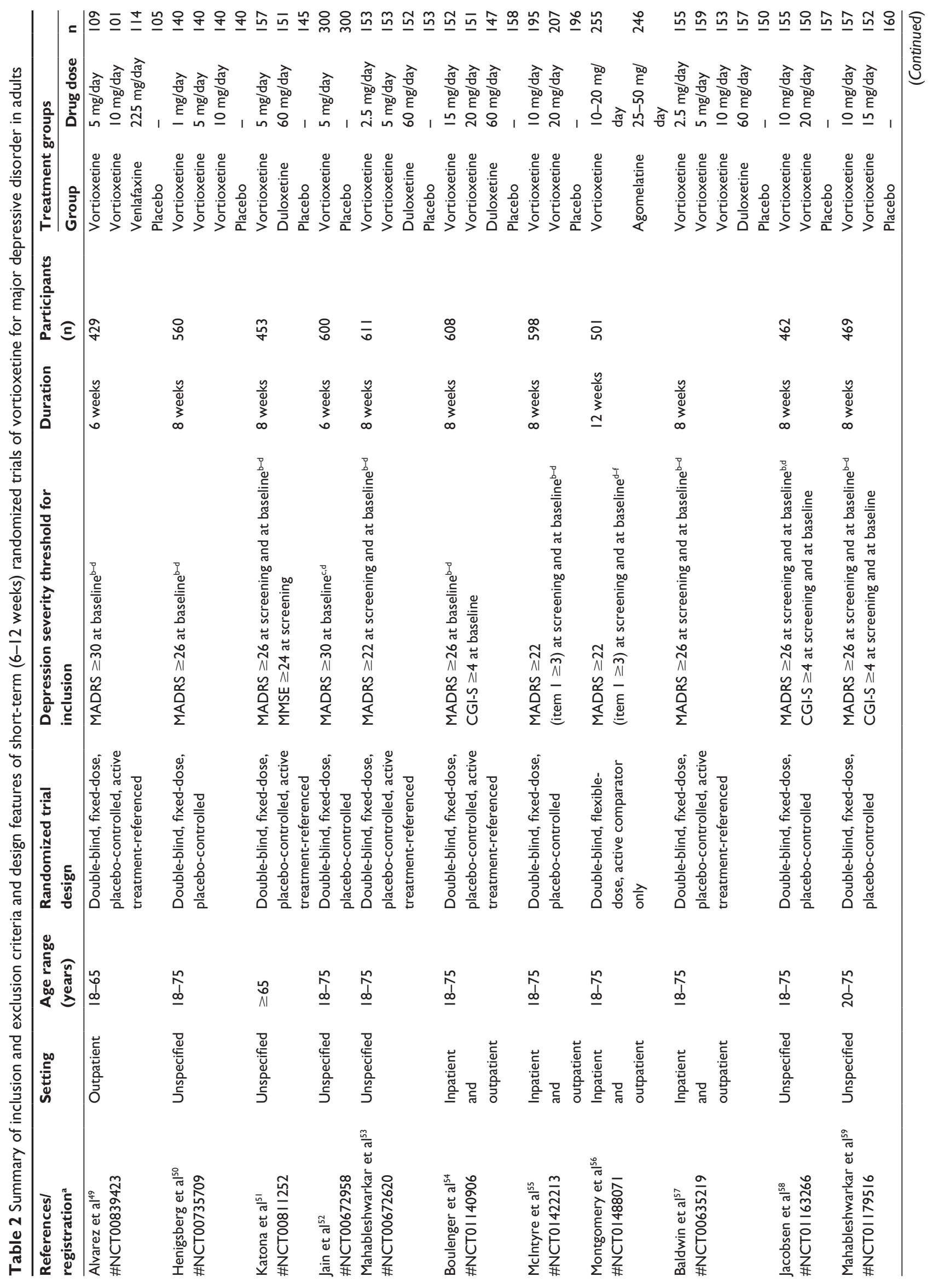



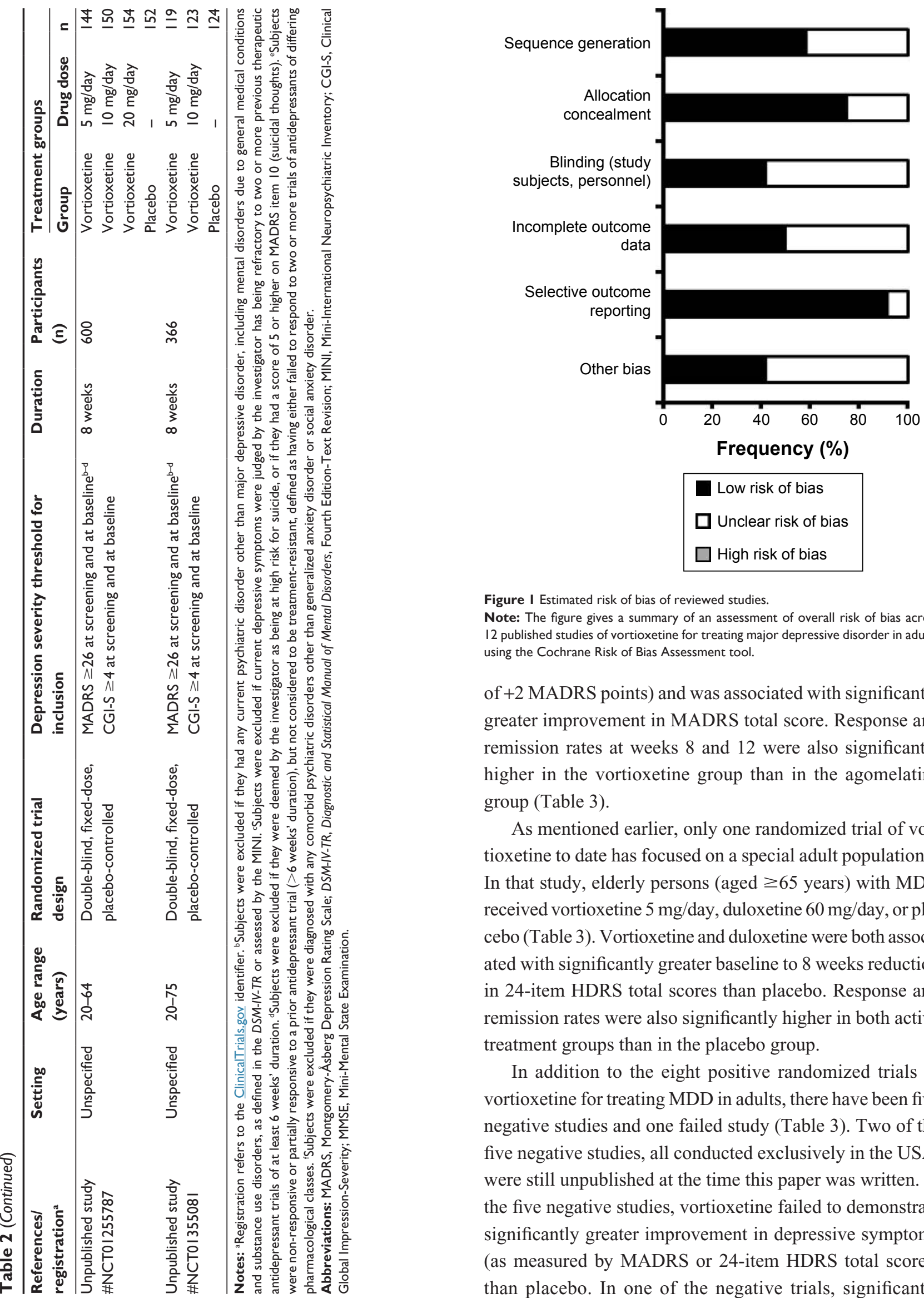

Low risk of bias

Unclear risk of bias

High risk of bias

Figure I Estimated risk of bias of reviewed studies.

Note: The figure gives a summary of an assessment of overall risk of bias across 12 published studies of vortioxetine for treating major depressive disorder in adults, using the Cochrane Risk of Bias Assessment tool.

of +2 MADRS points) and was associated with significantly greater improvement in MADRS total score. Response and remission rates at weeks 8 and 12 were also significantly higher in the vortioxetine group than in the agomelatine group (Table 3).

As mentioned earlier, only one randomized trial of vortioxetine to date has focused on a special adult population. ${ }^{51}$ In that study, elderly persons (aged $\geq 65$ years) with MDD received vortioxetine $5 \mathrm{mg} /$ day, duloxetine $60 \mathrm{mg}$ /day, or placebo (Table 3). Vortioxetine and duloxetine were both associated with significantly greater baseline to 8 weeks reduction in 24-item HDRS total scores than placebo. Response and remission rates were also significantly higher in both active treatment groups than in the placebo group.

In addition to the eight positive randomized trials of vortioxetine for treating MDD in adults, there have been five negative studies and one failed study (Table 3). Two of the five negative studies, all conducted exclusively in the USA, were still unpublished at the time this paper was written. In the five negative studies, vortioxetine failed to demonstrate significantly greater improvement in depressive symptoms (as measured by MADRS or 24-item HDRS total scores) than placebo. In one of the negative trials, significantly 


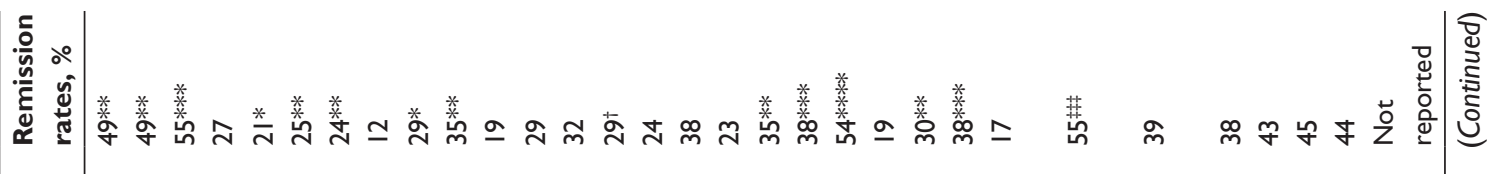

ڤั̀

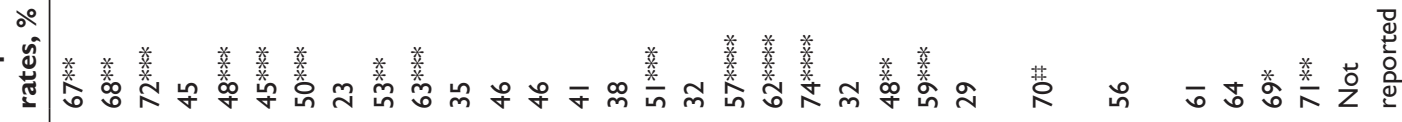

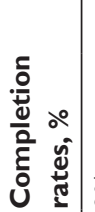

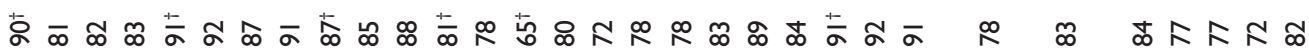

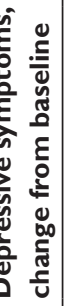

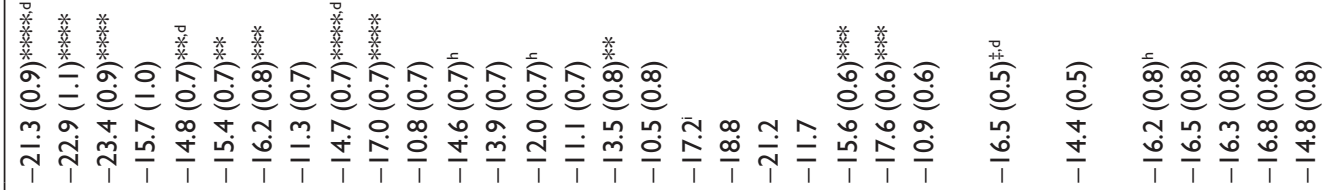




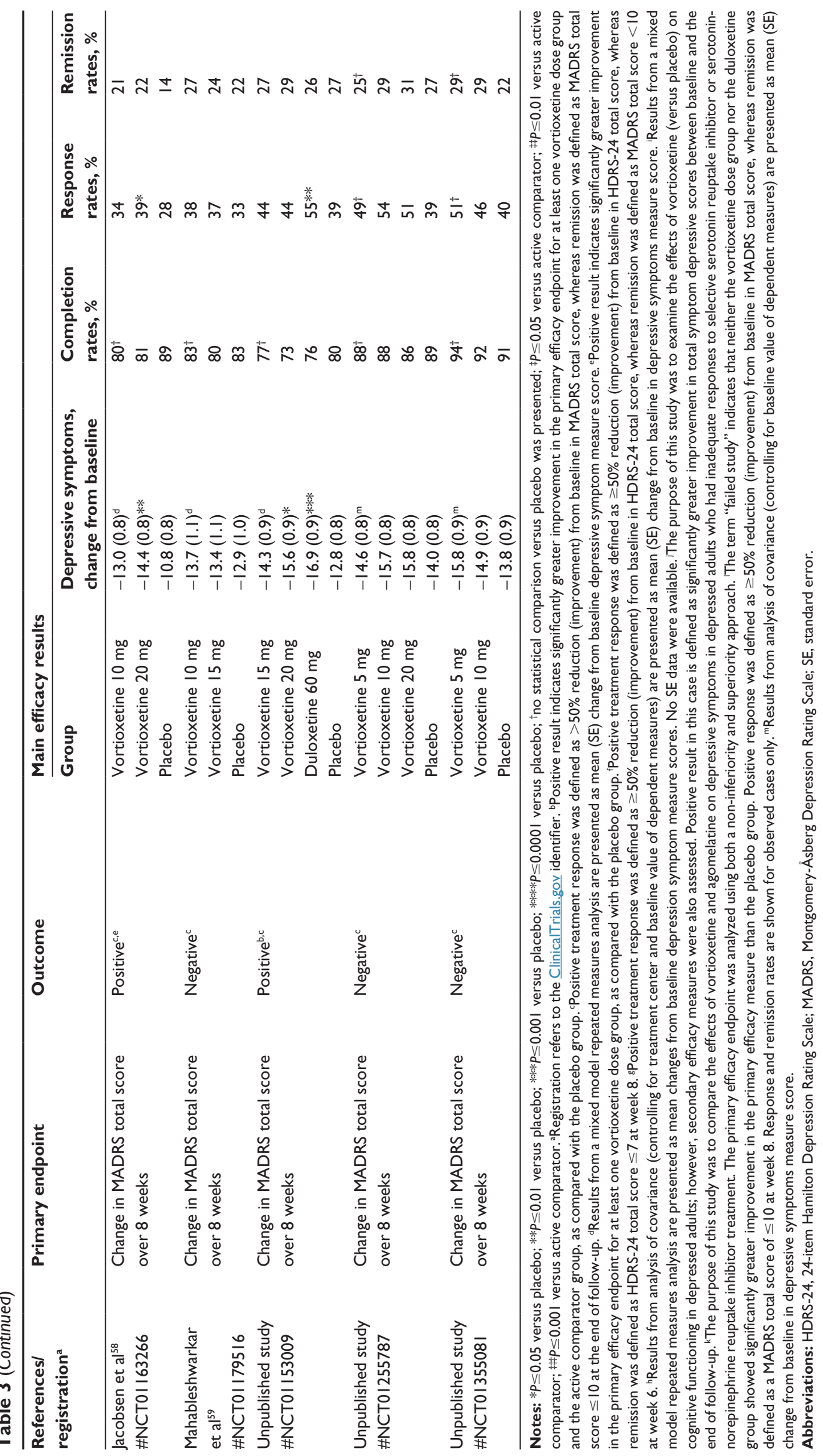


greater improvement in depressive symptoms was observed in an active control group that received duloxetine (60 mg daily). ${ }^{53}$

Study completion rates in the negative studies were generally comparable with completion rates in the positive trials; however, response and remission rates in the negative trials ranged from $38 \%$ to $51 \%$ and from $24 \%$ to $31 \%$, respectively, with vortioxetine and were therefore lower than those observed in the positive trials. Placebo response and remission rates, on the other hand, were generally comparable between negative trials (32\%-46\% and $22 \%-32 \%$, respectively) and positive trials. In the failed study, none of the active treatment groups, including those treated with duloxetine, showed significantly greater reduction (versus placebo) in MADRS total scores, the primary efficacy endpoint, between baseline and 8 weeks. ${ }^{57}$ Rates of positive treatment response were significantly higher with vortioxetine $10 \mathrm{mg}$ and duloxetine $60 \mathrm{mg}$, compared with placebo; however, none of the active treatment groups demonstrated significantly higher rates of remission than did placebo. The range of vortioxetine doses in the negative and failed studies (2.5-20 mg daily) overlapped almost entirely with the vortioxetine dose range in the positive studies (5-20 mg daily), making it difficult to clearly identify dose-response effects on depressive symptoms or on rates of response and remission.

\section{Pooled data analyses from acute-phase studies}

Two meta-analyses of randomized double-blind trials summarized the acute-phase efficacy of vortioxetine across its entire dose range, and thus afforded the opportunity to determine more precise estimates of vortioxetine efficacy and rates of response and remission, given the generally positive but ultimately mixed results from short-term trials. Both meta-analyses showed significantly higher rates of response and remission and greater reduction in depressive symptoms than placebo. The first meta-analysis of seven heterogeneous studies (3,934 randomized subjects) showed significantly greater reduction in MADRS total score from baseline with vortioxetine, as compared with placebo (weighted mean difference $-3.9,95 \%$ confidence interval $[\mathrm{CI}]-2.3$ to -2.6$){ }^{62}$ Pooled response data showed significantly higher odds of a positive treatment response with vortioxetine than placebo (odds ratio [OR] 2.9, 95\% CI 2.4-3.4). Despite significant heterogeneity among the seven reviewed studies, the weighted mean difference in MADRS total scores from baseline did not change significantly in sensitivity analyses that excluded individual studies sequentially, indicating that no single study had too great an impact on results from the overall analysis. There was no significant effect of treatment duration on antidepressive efficacy. The interpretation of this meta-analysis was limited by inclusion of only published studies. The risk of publication bias was left unaddressed, and only a limited number of moderators of study outcome were explored. A more recent meta-analysis of eleven heterogeneous randomized trials (4,947 subjects with MDD) included both published and unpublished studies, and also showed a smaller but statistically significant reduction from baseline in depressive symptoms, compared with placebo (standardized mean difference [SMD] $-0.2,95 \% \mathrm{CI}-0.3$ to -0.1$).{ }^{63}$ Pooled response and remission rates were significantly higher with vortioxetine than with placebo (positive treatment response, OR 1.7, 95\% CI 1.3-2.1; remission, OR 1.4, 95\% CI 1.1-1.8). The larger sample size of this meta-analysis permitted exploration of a greater number of possible moderators of study outcome. No single study appeared to have too great an impact on the results from the overall analysis, as was the case with the first meta-analysis. Interestingly, meta-regression analyses showed that studies conducted outside of the USA (or in mixed locations) demonstrated significantly more favorable odds of response and remission for vortioxetine than studies conducted exclusively in the USA. Subgroup analyses of US studies showed significantly higher odds of response (OR 1.22, 95\% CI 1.02-1.45), but not remission, as compared with placebo. Other moderators, such as publication status and study location, did not appear to have a consistent effect on primary or secondary efficacy outcomes between vortioxetine and placebo.

Pooled analyses of randomized trial data also permitted indirect examination of the comparative antidepressive effects of vortioxetine, relative to other antidepressants. As mentioned earlier, only one randomized trial in depressed adults was designed to directly compare the antidepressive effects of vortioxetine and another antidepressant (agomelatine). ${ }^{56}$ The meta-analysis of the eleven randomized trials reviewed earlier included seven studies (2,843 randomized subjects) that involved active control groups treated with venlafaxine or duloxetine. ${ }^{63}$ When the analysis was restricted to these seven studies, there were no significant differences in standardized mean reduction from baseline in depressive symptoms (SMD 0.08, 95\% CI -0.06 to 0.22 ) or odds of positive treatment response (OR $0.8,95 \%$ CI $0.6-1.1$ ) or remission (OR $0.8,95 \%$ CI 0.6-1.2) between vortioxetine and other antidepressants. However, meta-regression analyses that excluded the study comparing the efficacy of vortioxetine and agomelatine showed that the pooled effect estimates 
for SMD in depression severity and rates of response and remission favored SNRIs (venlafaxine, duloxetine) over vortioxetine. These results were consistent with those of a pooled analysis of data from five short-term (6- to 8-week) randomized trials of vortioxetine for MDD in adults that compared effect sizes for treatment response and remission with vortioxetine versus venlafaxine and duloxetine. ${ }^{64}$ The number needed to treat (NNT) for a positive treatment response (versus placebo) was lower (better) with both venlafaxine (NNT 3 ) and duloxetine (NNT 5) than with vortioxetine (NNT 7). Effect sizes for remission were also more robust for venlafaxine (NNT 4) and duloxetine (NNT 6) than for vortioxetine (NNT 11). The only other source of comparative acute-phase efficacy data between vortioxetine and other antidepressants comes from a recently published meta-regression analysis that attempted to examine the comparative efficacy of vortioxetine and six other antidepressants using data from 57 short-term (up to 8 weeks) randomized trials of adults with MDD. ${ }^{65}$ The meta-regression analysis showed comparable efficacy of vortioxetine with agomelatine (SMD -0.16 , $P=0.11$ ), desvenlafaxine (SMD 0.03, $P=0.80$ ), duloxetine (SMD 0.09, $P=0.42$ ), escitalopram (SMD $-0.05, P=0.70$ ), sertraline (SMD $-0.04, P=0.83)$, venlafaxine (SMD 0.12, $P=0.33$ ), and vilazodone (SMD $-0.25, P=0.11$ ). Comparative efficacy estimates from this analysis, however, were based primarily on indirect comparisons between vortioxetine and other antidepressants, rather than direct comparisons between agents in single randomized trials.

The identification of dose-response effects of vortioxetine on depressive symptoms has been challenging because of the near-complete overlap of vortioxetine dose ranges between the positive studies and the failed/negative studies (Table 3 ), and inconsistent dose effects in individual studies and pooled analyses of data from short-term randomized trials. For example, the first reviewed meta-analysis of seven randomized trials quantified the effects of vortioxetine on depressive symptoms across its entire dose range, and a meta-regression analysis of that data showed significantly greater reductions in MADRS total scores in patients who received higher vortioxetine doses. ${ }^{62}$ On the other hand, vortioxetine $5 \mathrm{mg} /$ day was associated with significantly greater improvement from baseline in 24-item HDRS total score than placebo (SMD 3.0 , 95\% CI 1.2-4.8, where values greater than 0 indicate superiority of vortioxetine to placebo) in a meta-analysis of five heterogeneous randomized trials with data analyses restricted to 1,700 subjects randomized to vortioxetine $5 \mathrm{mg}$ / day or placebo. ${ }^{66}$ Moreover, a systematic review of eleven short-term randomized trials of vortioxetine for MDD in adults showed that the largest effect size for positive treatment response was observed for vortioxetine $15 \mathrm{mg} /$ day (NNT 6), while the largest effect size for remission was observed with vortioxetine $5 \mathrm{mg}$ /day (NNT 8). ${ }^{64}$

\section{Longer-term (maintenance) treatment of major depression}

There is a paucity of efficacy data from longer-term studies of adults treated with vortioxetine (Table 4). Nearly all longerterm studies were open-label extensions of acute-phase efficacy trials in which eligible participants who completed the acute-phase studies received flexibly dosed vortioxetine (2.5-20 mg daily) for an additional 52 weeks. Only two of these studies have been published to date. ${ }^{67,68}$ In general, the results of these extension-phase studies suggest that antidepressive efficacy is maintained for up to 52 weeks in persons who completed the acute-phase studies; however, dropout rates were high during the 52-week extension phases.

In a more rigorous, randomized relapse prevention study in adults with MDD, 639 patients received 12 weeks of open-label, flexible-dose treatment with vortioxetine (5-10 mg/day) ${ }^{69}$ Of these, 400 patients who achieved symptom remission (MADRS total score $\leq 10$ at weeks 10 and 12) were randomized to double-blind treatment with vortioxetine (at fixed doses of 5 or $10 \mathrm{mg} /$ day) or to placebo, and were followed for up to 64 weeks. A significantly higher risk of MDD relapse was observed in the placebo group than in the vortioxetine group within the first 24 weeks of doubleblind follow-up (hazard ratio 2.01, 95\% CI 1.26-3.21), after adjusting for the effects of age, sex, race, study center, country, MADRS total scores at baseline and at randomization, and body weight at baseline and after randomization. Vortioxetine was associated with a significantly longer time to MDD relapse than placebo during the same time interval $(P=0.0035)$.

\section{Safety and tolerability Pooled analyses of data from short-term randomized trials}

Three meta-analyses of short-term randomized trials also summarized data on the safety and tolerability of vortioxetine in the treatment of adults with MDD. ${ }^{62,63,66}$ In general, these meta-analyses reported a higher incidence of treatmentemergent adverse effects and higher rates of discontinuation owing to adverse effects with vortioxetine than with placebo. One meta-analysis also investigated rates of treatment discontinuation with vortioxetine, as compared with active control treatment with venlafaxine $(225 \mathrm{mg} /$ day $)$ or duloxetine 


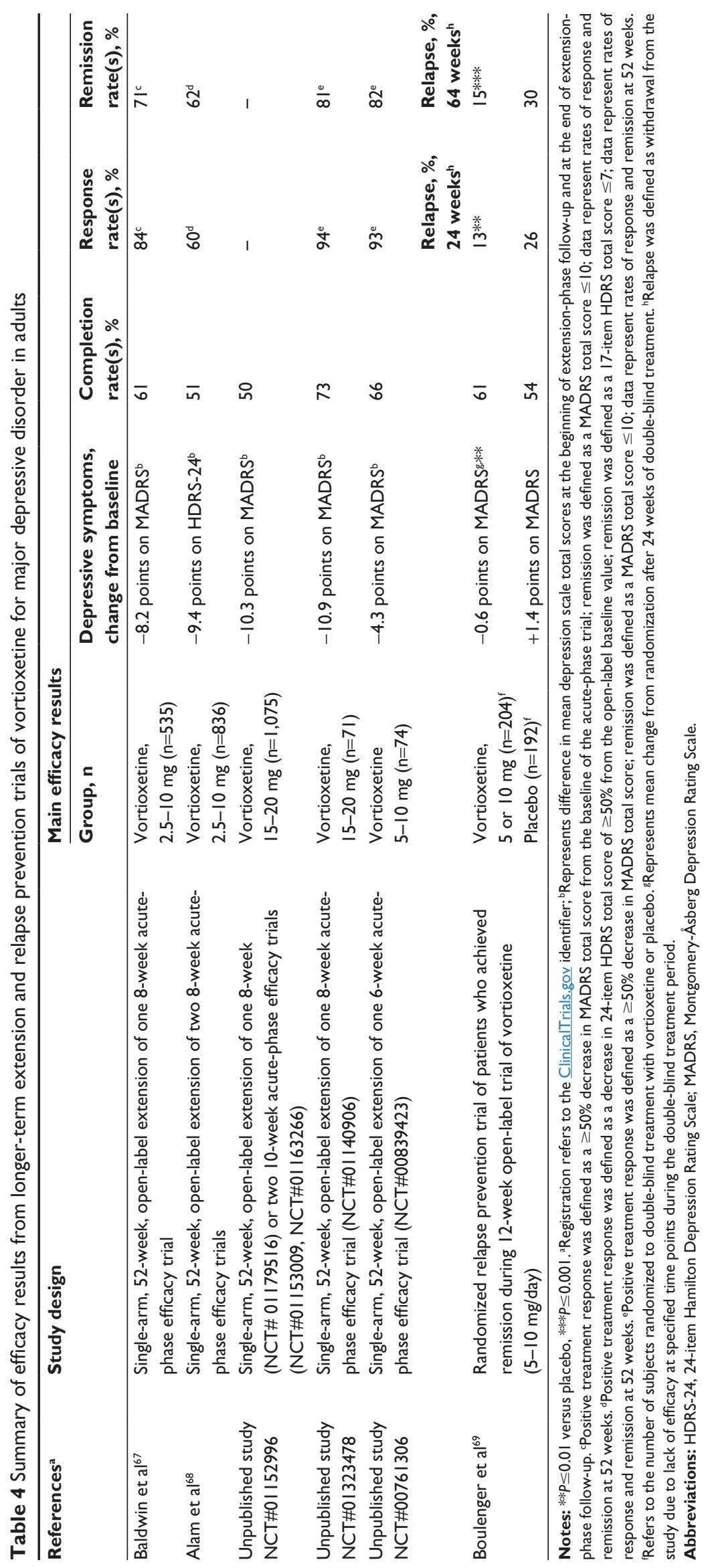


(60 mg/day), from seven heterogeneous randomized trials (involving 3,111 randomized subjects). ${ }^{63}$ Vortioxetine was associated with significantly lower rates of discontinuation owing to adverse effects, as compared with active controls (OR 0.73, 95\% CI 0.55-0.96). Dose-dependent effects of vortioxetine on the incidence of (or treatment discontinuation due to) adverse effects were not reported in these studies.

Another pooled data analysis calculated the number needed to harm $(\mathrm{NNH})$ for treatment discontinuation owing to adverse effects according to vortioxetine dose (versus placebo) in eleven short-term (up to 8 weeks) randomized trials. ${ }^{64}$ The NNH for treatment discontinuation owing to adverse effects with vortioxetine was 22 at $20 \mathrm{mg}$ /day, 24 at $15 \mathrm{mg} /$ day, and 40 at $10 \mathrm{mg} /$ day. $\mathrm{NNH}$ values for vortioxetine were generally higher (more favorable) than corresponding $\mathrm{NNH}$ values for active comparator antidepressants in the pooled data analysis, ie, NNH 10 with venlafaxine $225 \mathrm{mg}$ /day and 20 with duloxetine $60 \mathrm{mg}$ /day. NNH values were not statistically significant for vortioxetine (versus placebo) at doses $\leq 5 \mathrm{mg}$ /day.

Pooled randomized trial data addressing the incidence of specific adverse effects are provided in the vortioxetine drug label (Figure 2), which summarizes safety data from 4,746 patients (aged 19-88 years) with MDD enrolled in short-term randomized trials..$^{45}$ Of these, 2,616 received treatment with vortioxetine (5-20 mg/day) over 6-8 weeks.

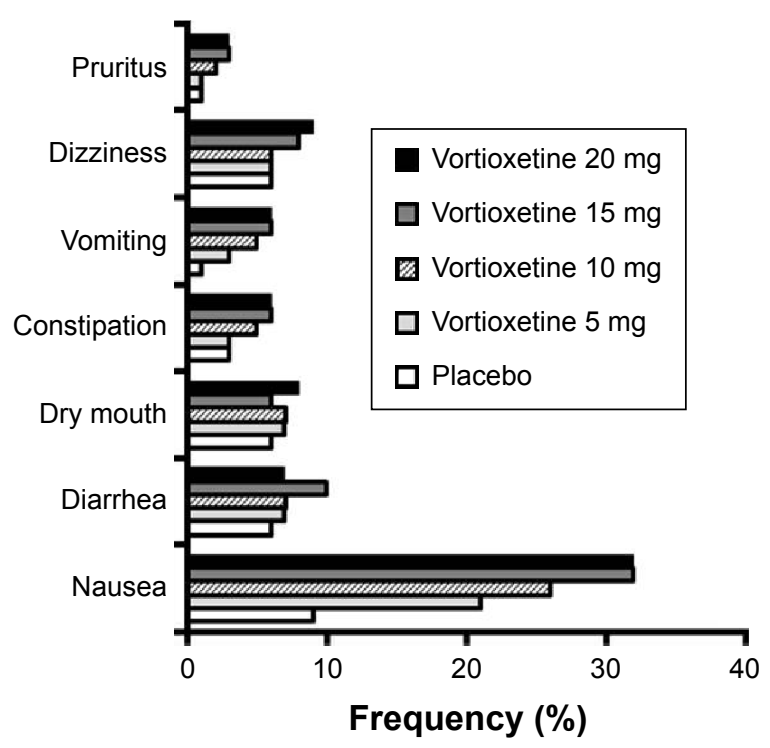

Figure 2 Frequency of selected treatment-emergent adverse effects, based on pooled data from short-term randomized trials of vortioxetine in adults with major depression.

Notes: This graph displays the pooled frequency of selected treatment-emergent adverse effects by vortioxetine dose from short-term (6-8 weeks) placebo-controlled studies in adults with major depression (2,616 were treated with vortioxetine), as reported in the vortioxetine drug label. Only selected adverse effects that occurred in $\geq 2 \%$ of vortioxetine-treated patients at any dose and occurred $\geq 2 \%$ more frequently with vortioxetine than with placebo are shown.

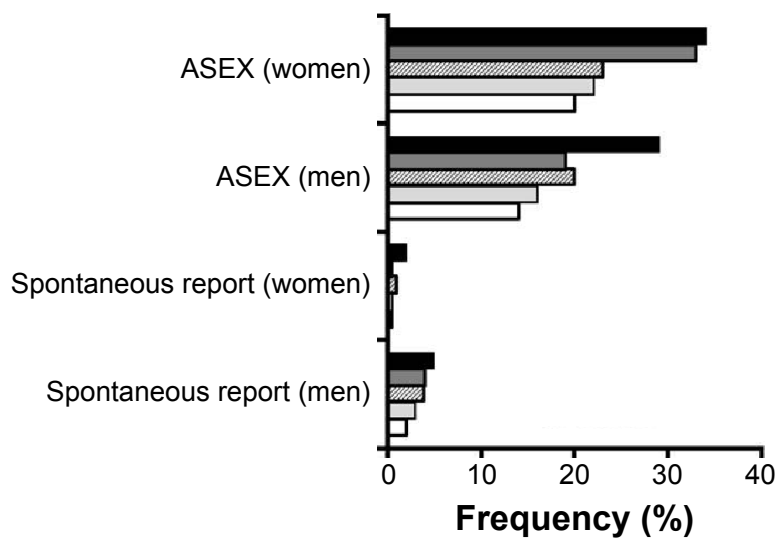

$\square$ Vortioxetine $20 \mathrm{mg}$
$\square$ Vortioxetine $15 \mathrm{mg}$
Vortioxetine $10 \mathrm{mg}$
$\square$ Vortioxetine $5 \mathrm{mg}$
$\square$ Placebo

Figure 3 Frequency of selected adverse effects on sexual functioning, based on pooled data from short-term randomized trials of vortioxetine in adults with major depression.

Notes: This graph displays the pooled sex-specific frequencies of treatment-emergent adverse effects on sexual performance by spontaneous self-report and according to Arizona Sexual Experiences Scale scores, as reported in the vortioxetine drug label. Abbreviation: ASEX, Arizona Sexual Experiences Scale.

The most common adverse effect attributable to vortioxetine treatment was nausea, the frequency of which increased in a dose-dependent manner from $21 \%$ at $5 \mathrm{mg} /$ day to $32 \%$ at $15 \mathrm{mg}$ and $20 \mathrm{mg} /$ day, and was substantially higher than that on placebo (9\%) across all vortioxetine doses (Figure 1). Although treatment-emergent nausea tended to be mild or moderate in intensity and persisted for a median duration of 2 weeks, it was still the most common adverse effect leading to vortioxetine discontinuation. Other commonly reported adverse effects across vortioxetine doses included diarrhea (7\%-10\%), dizziness (6\%-9\%), vomiting (3\%-6\%), dry mouth $(6 \%-8 \%)$, constipation $(3 \%-6 \%)$, and pruritus (1\%-3\%). With the exception of nausea, vomiting, and constipation, the incidence rates for these adverse effects were comparable between vortioxetine and placebo.

\section{Safety data from longer-term studies}

Longer-term safety data for vortioxetine were reported in two published 52-week open-label extension studies and one randomized, double-blind relapse prevention study. ${ }^{67-69}$ In both open-label extension studies, the most commonly reported individual adverse effects during open-label follow-up were nausea $(15 \%-72 \%)$, headache $(13 \%-20 \%)$, and nasopharyngitis $(10 \%-15 \%))^{67,68}$ In the first open-label study, a total of 18 patients reported one or more serious adverse 
events, five of which involved reported suicidal behavior or self-harm. ${ }^{67}$ Two deaths occurred during follow-up, neither of which was attributed to the effects of vortioxetine treatment. In the second open-label study, 38 serious adverse events, five of which were adjudicated as being possibly related to vortioxetine (depression, major depression, supraventricular tachycardia, paroxysmal tachycardia, and left hemispheric ischemic stroke). ${ }^{6}$ Three subjects with serious adverse events (depression, major depression, left hemispheric ischemic stroke) withdrew from the study. One suicide attempt and two cases of suicidal ideation were reported, but these events were not considered to be study drug-related. In both openlabel studies, there were no clinically significant changes in vital signs, electrocardiogram parameters, or clinical laboratory values.

In the randomized relapse prevention trial, nausea (25.7\%), headache $(18.3 \%)$, and nasopharyngitis $(8.1 \%)$ were the most commonly reported adverse effects during the initial open-label, flexible-dose treatment phase. ${ }^{69}$ During the 24-week, double-blind treatment phase, a significantly higher proportion of vortioxetine-treated subjects experienced nausea than did those randomized to placebo (8.8\% versus $3.1 \%, P<0.05)$. There were no statistically significant differences in the occurrence of other adverse effects, including headache and nasopharyngitis, between patients randomized to vortioxetine continuation treatment and those randomized to placebo. A numerically higher proportion of patients randomized to vortioxetine discontinued treatment during the double-blind phase owing to adverse effects $(7.8 \%)$ as compared with placebo $(2.6 \%)$. A total of seven patients in the vortioxetine group and four patients in the placebo group reported serious adverse events in the double-blind treatment period; no serious adverse events were reported by more than one patient. There were two deaths by suicide that occurred during the screening period. There were no clinically significant changes in vital signs, electrocardiogram parameters, or clinical laboratory values during either the open-label lead-in or double-blind relapse prevention periods of the study.

\section{Treatment-emergent sexual dysfunction}

Treatment-emergent sexual dysfunction is reviewed separately given how frequently it leads to poor adherence to antidepressants. ${ }^{70-72}$ A summary of pooled data on selfreported adverse effects of vortioxetine on sexual desire, performance, and satisfaction, as well as systematic measurement of sexual effects of treatment using the Arizona Sexual Experiences Scale (ASEX) ${ }^{73}$ has been provided in the vortioxetine drug label (Figure 3). ${ }^{45}$ For men, the incidence of self-reported adverse effects related to sexual dysfunction while being treated with vortioxetine ranged from 3\% at $5 \mathrm{mg} /$ day to $5 \%$ at $20 \mathrm{mg}$ /day, compared with $2 \%$ for placebo. For women, the incidence of self-reported sexual adverse effects with vortioxetine ranged from $<1 \%$ at $5 \mathrm{mg} /$ day to $2 \%$ at $20 \mathrm{mg} /$ day, compared with $<1 \%$ for placebo.

For the pooled data analysis in the vortioxetine drug label, ${ }^{45}$ subjects were also classified as having incident sexual dysfunction if, during two consecutive study visits, they had either an ASEX total score $\geq 19$, a single ASEX item score $\geq 5$ (in any one of five items that rated sex drive, ease of arousal, ability to achieve erections [men] or lubrication [women], ease of achieving orgasm, and orgasmic satisfaction on a scale of 1-6), or three or more ASEX items with a score $\geq 4$. Using this more sensitive definition, incidence rates of sexual dysfunction on vortioxetine ranged from $16 \%$ at $5 \mathrm{mg}$ /day to $29 \%$ at $20 \mathrm{mg} /$ day (compared with $14 \%$ with placebo) in men, and from $22 \%$ at $5 \mathrm{mg}$ /day to $34 \%$ at 20 $\mathrm{mg} /$ day (compared with $20 \%$ for placebo) in women. Additional pooled analyses of the same data showed that the $\mathrm{NNH}$ for incident sexual dysfunction (versus placebo) decreased with increasing vortioxetine dose, ranging from 50 with vortioxetine $5 \mathrm{mg}$ /day to 7 with vortioxetine $20 \mathrm{mg}$ /day) in men. In women, corresponding NNH values ranged from 50 with vortioxetine $5 \mathrm{mg}$ /day to 8 with vortioxetine $20 \mathrm{mg} /$ day.

Comparative risks of sexual adverse effects between vortioxetine and the venlafaxine and duloxetine comparator groups from short-term randomized trials was not provided in the vortioxetine drug label. Based on a pooled analysis of data from two short-term randomized trials, the estimated $\mathrm{NNH}$ for treatment-emergent sexual dysfunction was 10 with venlafaxine and 8 with duloxetine. ${ }^{64}$ Corresponding $\mathrm{NNH}$ values for spontaneously reported adverse effects related to sexual dysfunction with vortioxetine (versus placebo) ranged from 100 (5 mg/day) to 34 (20 mg/day) in men. For women, estimated NNH values for spontaneously reported adverse effects related to sexual dysfunction with vortioxetine exceeded 100 for all vortioxetine dose groups.

\section{Changes in body weight}

Vortioxetine treatment was not associated with significant changes in body weight during short-term (6- to 8-week) randomized trials in adults with MDD, as reported in the drug label. ${ }^{45}$ Furthermore, in a pooled analysis of eleven short-term trials, clinically significant weight gain (defined as an increase in body weight from baseline of $\geq 7 \%$ ) 
occurred infrequently (incidence $<3 \%$ ) in the vortioxetine dose groups, ranging from $1 \mathrm{mg} /$ day to $10 \mathrm{mg} / \mathrm{day}$, and no subjects discontinued treatment owing to weight gain. Given the brief follow-up period of these registration-type studies, anthropometric data from longer-term studies are perhaps more informative. Mean changes in body weight were modest $(0.7-1.1 \mathrm{~kg})$ over 52 weeks in the two published open-label extension studies. ${ }^{67,68}$ In the randomized relapse prevention study, there were no clinically relevant changes in body weight during either the open-label or double-blind study periods. ${ }^{69}$

\section{Discontinuation syndrome symptoms}

A constellation of distressing physical and psychological symptoms emerging within the first few days after abrupt discontinuation or rapid dose de-escalation of several antidepressants has been collectively referred to as antidepressant discontinuation syndrome. ${ }^{74-76}$ The incidence of discontinuation syndrome symptoms after abrupt cessation of vortioxetine treatment was evaluated in one published short-term (8-week) randomized trial, ${ }^{54}$ two unpublished short-term randomized trials (NCT01153009, NCT01163266), and one longer-term relapse prevention trial. ${ }^{69}$ Discontinuation syndrome symptoms were identified in the short-term randomized trials by using the clinician-rated Discontinuation Emergent Signs and Symptoms (DESS) checklist. ${ }^{77}$

In the published randomized trial, those who completed 8 weeks of double-blind follow-up on vortioxetine $15 \mathrm{mg} /$ day, vortioxetine $20 \mathrm{mg} /$ day, duloxetine $60 \mathrm{mg} /$ day, or placebo were evaluated with the DESS at week 8 (to obtain a baseline value). ${ }^{54}$ Afterward, vortioxetine-treated patients were rapidly switched to placebo, while those who received placebo remained on placebo. Duloxetine-treated patients were tapered to $30 \mathrm{mg}$ /day during week 9, and were then switched to placebo. After study completion, patients were reassessed using the DESS once weekly over the next 2 weeks (weeks 9 and 10). There were no significant between-group differences in mean DESS total score at week 8. At week 9, mean DESS total scores increased numerically from baseline and were numerically greater than placebo in all active treatment groups. At week 10, there were no longer statistically significant differences in mean DESS total score between either of the vortioxetine dose groups and placebo. On the other hand, there was a large and statistically significant increase in DESS total score from baseline to week 10 in the duloxetine group (0.5 to $2.8, P<0.0001)$ after switching to placebo. Mean DESS total score at week 10 was significantly higher in the duloxetine group than placebo $(P<0.001)$.
The two unpublished randomized trials evaluated change in DESS scores after discontinuation of vortioxetine at daily doses of 10, 15, or $20 \mathrm{mg}$. However, specific information on study drug discontinuation procedures and mean DESS scores after discontinuation of vortioxetine was not available on the ClinicalTrials.gov website, or in published online abstracts. ${ }^{78,79}$ Both abstracts report placebo-like DESS scores associated with discontinuation of vortioxetine treatment, but no empirical data were presented.

In the randomized relapse prevention study, potential discontinuation syndrome symptoms were assessed by spontaneous report during separate 2-week periods occurring at the end of the 12-week, open-label lead-in phase, and at the end of double-blind treatment. ${ }^{69}$ After both study periods were completed, vortioxetine treatment was abruptly discontinued. The authors reported that incidence rates of potential discontinuation syndrome symptoms after abrupt discontinuation of short-term and longer-term vortioxetine treatment were "at a placebo level". However, numerical data were not provided.

\section{Discussion}

\section{Summary of short-term efficacy data}

Vortioxetine is the most recently approved pharmacotherapy for MDD in adults. The therapeutic mechanism of action of vortioxetine is most likely linked to SERT blockade, a property shared by numerous other antidepressants, including the SSRIs, SNRIs, vilazodone, and most tricyclic antidepressants. ${ }^{80,81}$ The short-term (6- to 12-week) efficacy of vortioxetine for treating MDD in adults is supported by eight positive, randomized placebo-controlled trials. These short-term studies showed significantly greater reduction in depressive symptoms with vortioxetine at doses of 5-20 mg/ day, as compared with placebo. Our review of the individual trials suggested that antidepressive efficacy was most consistently observed at daily doses of 15-20 mg, but a dose-efficacy relationship with vortioxetine has not clearly emerged. Two unpublished positive studies showed superiority of vortioxetine (versus placebo) for reducing depressive symptoms only at the $20 \mathrm{mg} /$ day dose, and not at 10 or $15 \mathrm{mg} /$ day (NCT01153009, NCT 01163266). This observation of a possible dose-effect response is supported by the results of one meta-analysis of randomized trials, which showed significantly greater reduction in depressive symptoms among patients who received higher daily doses of vortioxetine. ${ }^{62}$ However, the most recent meta-analysis by Pae et al did not find a dose effect. ${ }^{63}$ Rates of positive treatment response and remission were consistently higher with vortioxetine than 
placebo in pooled analyses of randomized trial data, but a dose effect for achieving positive treatment response or remission was not demonstrated. ${ }^{64}$ Vortioxetine has been found to have similar efficacy for treating MDD in elderly patients as for younger adults in one randomized trial..$^{51}$

\section{How effective is vortioxetine?}

Vortioxetine is the latest agent to enter the competitive antidepressant market. Over 50 antidepressant drugs are available worldwide. The broad availability of such a large number of drugs for treating MDD in adults raises the question of what addition benefit vortioxetine will bring. Most marketed antidepressants (other than vilazodone or milnacipran) have been around for more than 10 years. Thus, many agents have an established place within the therapeutic armamentarium for treating MDD based on several years of clinical experience. Clinicians, patients, and caregivers will be looking for specific advantages for vortioxetine to offset higher cost as a new on-patent agent, in addition to a favorable adverse effect profile.

Although the results of short-term randomized trials have been mostly positive, only three studies were assessed as having low bias risk in all domains. ${ }^{49,51,56}$ The presence of bias in the other studies cannot be excluded, and there was insufficient methodological detail available for the three unpublished studies to permit a complete bias risk assessment. Furthermore, vortioxetine has not yet been consistently shown to improve measures of global functioning or quality of life, and high placebo response rates in many of the reviewed studies make it more difficult to estimate the clinical significance of vortioxetine effects on depressive symptoms. Differences in depressive symptom reduction between vortioxetine and placebo were assessed as being only small to medium in terms of effect size (SMD -0.22) in the most comprehensive meta-analysis of short-term trials. ${ }^{63}$ As noted by the authors, effect size differences may have been underestimated in the reviewed studies due to a variety of factors, including high placebo response rates. The clinical significance of vortioxetine efficacy data was more directly addressed in the systematic review and pooled data analysis that documented a NNT value of 7 for positive treatment response with vortioxetine (versus placebo) ${ }^{64}$ - a NNT value that exceeds the minimal clinically important difference threshold of 10 for antidepressant-placebo comparisons from randomized trials. ${ }^{82}$ How well this effect size difference generalizes to real-world patient care remains an open question. Indeed, there are well-known differences in the types of patients and the conditions under which antidepressant treatment take place in randomized trials as compared with routine clinical practice. ${ }^{83}$

\section{How does vortioxetine compare with other antidepressants?}

Currently, there is no evidence of clinical superiority for vortioxetine over other antidepressants. Many acute-phase, placebo-controlled studies included an active control group consisting of treatment with duloxetine or venlafaxine. However, individual trials were not sufficiently powered to provide valid comparisons other than with placebo. Pooled analyses of trials that included active comparator groups have provided some preliminary comparative efficacy data. The meta-analysis by Pae et al found no significant differences in depressive symptom reduction or odds of positive treatment response or remission between vortioxetine and a combined group consisting of all other antidepressants (SNRIs and agomelatine), ${ }^{63}$ although use of relatively modest doses of duloxetine $(60 \mathrm{mg} / \text { day })^{84,85}$ in five duloxetine-controlled studies may have introduced some bias toward null findings. Sharper contrasts in clinical effect sizes for antidepressive efficacy (versus placebo) between vortioxetine and SNRIs were drawn in a pooled analysis that showed generally lower (better) NNTs (versus placebo) for positive treatment response and remission with venlafaxine or duloxetine that with vortioxetine. These results raise the question of whether vortioxetine may have relatively weaker antidepressive effects than venlafaxine or duloxetine. However, it is important to note that no adequately powered comparative efficacy studies of vortioxetine and other antidepressants have been conducted with the exception of one randomized 12-week comparison of vortioxetine and agomelatine. ${ }^{56}$

There is also no current evidence of a more rapid onset of antidepressive effect with vortioxetine than with other antidepressants. In a review of short-term randomized trials conducted by the US Food and Drug Administration, there was some evidence of antidepressive efficacy after 2 weeks of treatment, with full antidepressive effects observed after 4-6 weeks of treatment. ${ }^{86}$ This is generally consistent with the expected time course to improvement with most first-line antidepressants. ${ }^{87,88}$

Future studies are now needed to establish specific roles for vortioxetine within the broader armamentarium of available antidepressant treatments. More studies are needed that directly compare the clinical effectiveness and safety of vortioxetine with commonly used antidepressants in depressed adults, and to determine the effectiveness of vortioxetine for adults with well-defined treatment-resistant MDD. 
The acute-phase studies in this review excluded patients with evidence of having failed two or more therapeutic trials of antidepressants for MDD, a common threshold for defining treatment resistance. ${ }^{89}$ No sequential treatment or switch studies (eg, from prior antidepressant treatments that were either poorly tolerated or did not result in adequate treatment response) have been published, with the exception of the vortioxetine-agomelatine comparison trial by Montgomery et al. ${ }^{56}$ Finally, no cost outcome data for vortioxetine treatment of MDD in adults are yet available, an important caveat for both patients and third-party payers considering the availability of older, less expensive alternatives to vortioxetine. At present, vortioxetine has been available for too brief a period of time to permit systematic evaluation of its impact on antidepressant prescribing patterns in mental health specialty and non-specialty practice.

\section{Clinical use of vortioxetine}

What then, based on available data, are the specific advantages of vortioxetine relative to other antidepressants that may inform clinical decision-making? The relatively longer half-life of vortioxetine facilitates once-daily dosing, and is a likely contributor to the low risk of antidepressant discontinuation syndrome symptoms after rapid discontinuation. At more clinically relevant doses of $10 \mathrm{mg} /$ day or higher, vortioxetine had more withdrawals owing to adverse effects than placebo, but generally less than activate comparators. No clinically relevant differences between vortioxetine and placebo were observed in terms of change in body weight, and very low rates of sedation or insomnia were reported in the reviewed studies. The most clinically significant adverse effect with vortioxetine treatment was dose-dependent nausea. The occurrence of treatment-emergent nausea is not unique to vortioxetine and is typically transient. ${ }^{28}$ Rates of spontaneously reported sexual dysfunction with vortioxetine treatment were nearly equivalent to that of placebo, and significantly lower than corresponding rates associated with venlafaxine and duloxetine treatment. ${ }^{49,51,67}$ This appears to be another potential advantage of vortioxetine over several other antidepressants; however, based on ASEX data from shortterm randomized trials, sexual dysfunction may be potentially treatment-limiting with vortioxetine at $20 \mathrm{mg} / \mathrm{day},{ }^{45}$ the highest approved dose. Finally, in addition to SERT blockade, vortioxetine interacts with numerous serotonin receptors. Although the clinical significance of most of these interactions is not firmly established for depressed patients, preclinical work has suggested that at least some of them may translate to therapeutic advantages beyond depressive symptoms (eg, sleep maintenance, cognitive functioning), and a lower incidence of vexing side effects (eg, sexual dysfunction). ${ }^{28,90}$

At what point might clinicians consider use of vortioxetine for treating their adult patients with MDD? As noted by Citrome,${ }^{64}$ the pharmacodynamic properties of vortioxetine are different enough from other antidepressant monotherapies to suggest that a therapeutic trial of vortioxetine could be considered if the clinical response to other antidepressants is inadequate, or if they are poorly tolerated. Vortioxetine can be given stronger consideration for patients in whom antidepressant therapy is required but sexual side effects or sedation has resulted in premature treatment discontinuation. Vortioxetine is currently under development for treating generalized anxiety disorder, ${ }^{91}$ a common anxiety syndrome that frequently co-occurs with MDD. ${ }^{92,93}$ Results of published short-term randomized trials of vortioxetine (2.5-10 mg/day) for treating generalized anxiety disorder have been mixed. ${ }^{94-96}$ However, if shown to be efficacious at higher doses in future studies, vortioxetine may be a viable treatment option for patients with comorbid MDD and generalized anxiety disorder who do not respond adequately to psychosocial and/or other antidepressant treatments. Importantly, vortioxetine appears to be effective for treating symptoms of MDD in the elderly based on the results of one randomized trial for which recruitment was focused on this specific population. ${ }^{51}$ Additional studies in geriatric patients with MDD are needed to confirm these positive results. Still, for depressed elderly patients, the apparent lack of sedation, changes in body weight, worrisome changes in vital signs, and electrocardiogram parameters may be particularly attractive features of vortioxetine. Vortioxetine does not appear to adversely affect psychomotor or cognitive performance, ${ }^{51,55,97}$ which may translate into additional safety advantages when treating older patients with vortioxetine, as compared with more sedating antidepressant drugs.

\section{Limitations and future directions}

We did not conduct a systematic review of studies of vortioxetine efficacy for treating MDD in adults. As such, our conclusions must be interpreted with the caveats applied to non-systematic literature reviews including lack of a prior protocol, lack of focused review questions with a specific electronic literature search strategy, and lack of well-defined inclusion/exclusion criteria for reviewed studies. This also applies to our review of pooled safety data, much of which was derived from the vortioxetine drug label and thus did not include a systematic account of methodological problems 
existing within and between studies. Additional limitations of this review reflect the limitations of the existing literature on vortioxetine effectiveness for treating MDD in adults. A large number of the reviewed studies were unpublished at the time of writing, and were not subjected to the rigors of peer review. All reviewed studies were supported by Takeda Pharmaceuticals Ltd, in conjunction with H Lundbeck A/S. As noted earlier, comparative effectiveness studies with older and more established antidepressants are lacking, and longterm pragmatic trials are needed in order to assess the effectiveness of vortioxetine under usual-care conditions. Clinical considerations for managing partial or incomplete responses to vortioxetine, eg, by increasing the daily dose, are limited by the lack of a clear relationship between vortioxetine dose and antidepressive benefit. It remains unclear what, if any, role there is for vortioxetine after failure of SSRIs or other antidepressants commonly utilized as first-line therapeutics. Until data from comparative effectiveness studies of vortioxetine and other marketed antidepressant drugs become available, the exact place of vortioxetine within the broader armamentarium of antidepressants will be very difficult to determine. Of course, for such studies to be conducted with sufficient validity to guide clinical practice, very large sample sizes with long-term follow-up will be required. These types of studies are logistically challenging and very expensive to conduct. Future research endeavors involving vortioxetine are thus likely to focus on broadening its therapeutic indications beyond major depression. More long-term data focused on maintenance of remission following acute-phase stabilization on vortioxetine treatment are needed, as are studies focused on vortioxetine effects on quality of life and functional capacity as primary endpoints.

\section{Conclusion}

Vortioxetine is the newest approved antidepressant treatment for MDD in adults. Positive results from short-term randomized trials have formed the basis for regulatory approval of vortioxetine for treating MDD in adults, but more long-term studies and comparative effectiveness trials are needed. Despite the lack of a clear dose-effect relationship, the existing acute-phase data provide some support for initiating vortioxetine at $10 \mathrm{mg}$ once daily, and increasing to 20 mg once daily as tolerated, for treating MDD in adults. Vortioxetine has a unique pharmacological profile that combines SERT blockade with direct occupancy of several serotonergic neuroreceptors. A number of theoretical advantages might be predicted from vortioxetine's pharmacodynamic activities, but the net effect of its interactions with serotonin receptors has not been adequately investigated. Vortioxetine has not been shown to be more rapidly or completely effective than other antidepressants. The low incidence of sexual side effects, treatment-emergent sedation, and drug-associated weight gain with vortioxetine, however, may be attractive for many patients.

\section{Disclosure}

WVB is supported by the Mayo Clinic Foundation. PEC is supported by the Mayo Clinic Foundation and the National Institute of Mental Health. The authors report no other conflicts of interest in this work.

\section{References}

1. World Health Organization. Depression. Fact sheet No.369, October 2012 Available from: www.cho.int/mediacentre/factsheets/fs369/en/. Accessed July 21, 2015.

2. American Psychiatric Association. Diagnostic and Statistical Manual of Mental Disorders, Fifth Edition. Washington, DC, USA: American Psychiatric Publishing; 2013.

3. World Health Organization. Promoting mental health: concepts, emerging evidence, practice (summary report). Geneva, Switzerland: World Health Organization; 2004. Available from: http:/www.who. int/mental_health/evidence/en/promoting_mhh.pdf. Accessed July 22, 2015.

4. Ferrari AJ, Charlson FJ, Norman RE. Burden of depressive disorders by country, sex, age, and year: findings from the Global Burden of Disease Study 2010. PLoS Med. 2013;10:e1001547.

5. World Health Organization and Department of Mental Health and Substance Abuse. Victorian Health Promotion Foundation. University of Melbourne. Promoting mental health: concepts, emerging evidence, practice: summary report. Geneva, Switzerland: World Health Organization; 2004. Available from: http://www.who.int/mental_health/ evidence/en/promoting_mhh.pdf. Accessed July 22, 2015.

6. Stegenga BT, Kamphuis MH, King M, Nazareth I, Geerlings MI. The natural course and outcome of major depressive disorder in primary care: the PREDICT-NL study. Soc Psychiatry Psychiatr Epidemiol. 2012;47:87-95.

7. Eaton WW, Shao H, Nestadt G, Lee HB, Bienvenu OJ, Zandi P. Population-based study of first onset and chronicity in major depressive disorder. Arch Gen Psychiatry. 2008;65:513-520.

8. Coryell W, Akiskal HS, Leon AC, et al. The time course of nonchronic major depressive disorder. Uniformity across episodes and samples. National Institute of Mental Health Collaborative Program on the Psychobiology of Depression - clinical studies. Arch Gen Psychiatry. 1994;51:405-410.

9. Mann JJ. The medical management of depression. $N$ Engl J Med. 2005;353:1819-1834.

10. National Institute for Clinical Excellence. Depression: management of depression in primary and secondary care. Clinical Guideline 23. London, UK: National Institute for Clinical Excellence; 2004. Available from: https://www.nice.org.uk/guidance/cg023. Accessed July 22, 2015.

11. Papakostas GI, Fava M. Does the probability of receiving placebo influence clinical trial outcome? A meta-regression of double-blind, randomized clinical trials in MDD. Eur Neuropsychopharmacol. 2009;19:34-40.

12. Shelton RC. Treatment options for refractory depression. J Clin Psychiatry. 1999;60 Suppl 4:57-61.

13. Keller MB. Past, present, and future directions for defining optimal treatment outcome in depression: remission and beyond. JAMA. 2003;289:3152-3160. 
14. Machado M, Iskedjian M, Ruiz I, Einarson TR. Remission, dropouts, and adverse drug reaction rates in major depressive disorder: a meta-analysis of head-to-head trials. Curr Med Res Opin. 2006;22:1825-1837.

15. Trivedi MH, Rush AJ, Wisniewski SR, et al. Evaluation of outcomes with citalopram for depression using measurement-based care in STAR*D: implications for clinical practice. Am J Psychiatry. 2006;163:28-40.

16. Rush AJ, Trivedi MH, Wisniewski SR, et al. Acute and longer-term outcomes in depressed outpatients requiring one or several treatment steps: a STAR*D report. Am J Psychiatry. 2006;163:1905-1917.

17. Thase ME, Friedman ES, Biggs MM, et al. Cognitive therapy versus medication in augmentation and switch strategies as second-step treatments: a STAR*D report. Am J Psychiatry. 2007;164:739-752.

18. Thase ME, Rush AJ. When at first you don't succeed: sequential strategies for antidepressant nonresponders. J Clin Psychiatry. 1997;58 Suppl 13: 23-29.

19. Trivedi MH, Fava M, Wisniewski SR, et al. Medication augmentation after the failure of SSRIs for depression. N Engl J Med. 2006;354: 1243-1252.

20. Papakostas GI, Shelton RC, Smith J, Fava M. Augmentation of antidepressants with atypical antipsychotic medications for treatmentresistant major depressive disorder: a meta-analysis. J Clin Psychiatry. 2007;68:826-831.

21. Vortioxetine Hydrobromide [database on the Internet]. National Center for Biotechnology Information. PubChem. Available from: http:// pubchem.ncbi.nlm.nih.gov/compound/Vortioxetine_hydrobromide. Accessed December 30, 2014.

22. Jans LA, Riedel WJ, Markus CR, Blokland A. Serotonergic vulnerability and depression: assumptions, experimental evidence and implications. Mol Psychiatry. 2007;12:522-543.

23. Schildkraut JJ. The catecholamine hypothesis of affective disorders: a review of supporting evidence. Am J Psychiatry. 1965;122:509-522.

24. Elhwuegi AS. Central monoamines and their role in major depression. Prog Neuropsychopharmacol Biol Psychiatry. 2004;28:435-451.

25. Bang-Andersen B, Ruhland T, Jorgensen M, et al. Discovery of 1-[2(2,4-dimethylphenylsulfanyl)phenyl]piperazine (Lu AA21004): a novel multimodal compound for the treatment of major depressive disorder. J Med Chem. 2011;54:3206-3221.

26. Arenberg J, Luntang-Jensen M, Sogaard B, Nilausen DO. Occupancy of the serotonin transporter after administration of Lu AA21004 and its relation to plasma concentration in healthy subjects. Basic Clin Pharmacol Toxicol. 2011;110:401-404.

27. Richelson E. Interactions of antidepressants with neurotransmitter transporters and receptors and their clinical relevance. J Clin Psychiatry. 2003;64 Suppl 13:5-12.

28. Sanchez C, Asin KE, Artigas F. Vortioxetine, a novel antidepressant with multimodal activity: review of preclinical and clinical data. Pharmacol Ther. 2015;145:43-57.

29. Mork A, Pehrson A, Brennum LT, et al. Pharmacological effects of Lu AA21004: a novel multimodal compound for the treatment of major depressive disorder. J Pharmacol Exp Ther. 2012;340:666-675.

30. Artigas F, Adell A, Celada P. Pindolol augmentation of antidepressant response. Curr Drug Targets. 2006;7:139-147.

31. Carr GV, Lucki I. The role of serotonin receptor subtypes in treating depression: a review of animal studies. Psychopharmacology (Berl). 2011;213:265-287.

32. Stahl SM. Mechanism of action of serotonin selective reuptake inhibitors. Serotonin receptors and pathways mediate therapeutic effects and side effects. J Affect Disord. 1998;51:215-235.

33. Pullar IA, Boot JR, Broadmore RJ, et al. The role of the 5-HT1D receptor as a presynaptic autoreceptor in the guinea pig. Eur J Pharmacol. 2004; 493:85-93.

34. Bonaventure P, Dugovic C, Kramer M, et al. Translational evaluation of JNJ-18038683, a 5-hydroxytryptamine type 7 receptor antagonist, on rapid eye movement sleep and in major depressive disorder. J Pharmacol Exp Ther. 2012;342:429-440.

35. Pehrson AL, Cremers T, Betry C, et al. Lu AA21004, a novel multimodal antidepressant, produces regionally selective increases of multiple neurotransmitters - a rat microdialysis and electrophysiology study. Eur Neuropsychopharmacol. 2013;23:133-145.
36. Blier P, Ward NM. Is there a role for 5-HT1A agonists in the treatment of depression? Biol Psychiatry. 2003;53:193-203.

37. Blier P, El Mansari M. Serotonin and beyond: therapeutics for major depression. Philos Trans R Soc Lond B Biol Sci. 2013;368:20120536.

38. Griebel G, Holmes A. 50 years of hurdles and hope in anxiolytic drug discovery. Nat Rev Drug Discov. 2013;12:667-687.

39. Kishi T, Meltzer HY, Matsuda Y, Iwata N. Azapirone 5-HT1A receptor partial agonist treatment for major depressive disorder: systematic review and meta-analysis. Psychol Med. 2014;44:2255-2269.

40. Portella MJ, de Diego-Adelino J, Ballesteros J, et al. Can we really accelerate and enhance the selective serotonin reuptake inhibitor antidepressant effect? A randomized clinical trial and a meta-analysis of pindolol in nonresistant depression. J Clin Psychiatry. 2011;72:962-969.

41. Berman RM, Anand A, Cappiello A, et al. The use of pindolol with fluoxetine in the treatment of major depression: final results from a doubleblind, placebo-controlled trial. Biol Psychiatry. 1999;45:1170-1177.

42. Berman RM, Darnell AM, Miller HL, Anand A, Charney DS. Effect of pindolol in hastening response to fluoxetine in the treatment of major depression: a double-blind, placebo-controlled trial. Am J Psychiatry. 1997; 154:37-43.

43. Perez V, Soler J, Puigdemont D, Alvarez E, Artigas F. A double-blind, randomized, placebo-controlled trial of pindolol augmentation in depressive patients resistant to serotonin reuptake inhibitors. Grup de Recerca en Trastorns Afectius. Arch Gen Psychiatry. 1999;56:375-379.

44. Perry EB, Berman RM, Sanacora G, Anand A, Lynch-Colonese K, Charney DS. Pindolol augmentation in depressed patients resistant to selective serotonin reuptake inhibitors: a double-blind, randomized, controlled trial. J Clin Psychiatry. 2004;65:238-243.

45. Brintellix (vortioxetine) package insert. Deerfield, IL, USA: Takeda Pharmaceuticals America, Inc; 2014.

46. Pearce EF, Murphy JA. Vortioxetine for the treatment of depression. Ann Pharmacother. 2014;48:758-765.

47. Hvenegaard MG, Bang-Andersen B, Pedersen H, Jorgensen M, Puschl A, Dalgaard L. Identification of the cytochrome P450 and other enzymes involved in the in vitro oxidative metabolism of a novel antidepressant, Lu AA21004. Drug Metab Dispos. 2012;40:1357-1365.

48. Chen G, Lee R, Hojer AM, Buchbjerg JK, Serenko M, Zhao Z. Pharmacokinetic drug interactions involving vortioxetine (Lu AA21004), a multimodal antidepressant. Clin Drug Investig. 2013;33:727-736.

49. Alvarez E, Perez V, Dragheim M, Loft H, Artigas F. A double-blind, randomized, placebo-controlled, active reference study of Lu AA21004 in patients with major depressive disorder. Int JNeuropsychopharmacol. 2012;15:589-600.

50. Henigsberg N, Mahableshwarkar AR, Jacobsen P, Chen Y, Thase ME. A randomized, double-blind, placebo-controlled 8-week trial of the efficacy and tolerability of multiple doses of Lu AA21004 in adults with major depressive disorder. J Clin Psychiatry. 2012;73: 953-959.

51. Katona C, Hansen T, Olsen CK. A randomized, double-blind, placebocontrolled, duloxetine-referenced, fixed-dose study comparing the efficacy and safety of Lu AA21004 in elderly patients with major depressive disorder. Int Clin Psychopharmacol. 2012;27:215-223.

52. Jain R, Mahableshwarkar AR, Jacobsen PL, Chen Y, Thase ME. A randomized, double-blind, placebo-controlled 6-wk trial of the efficacy and tolerability of $5 \mathrm{mg}$ vortioxetine in adults with major depressive disorder. Int J Neuropsychopharmacol. 2013;16:313-321.

53. Mahableshwarkar AR, Jacobsen PL, Chen Y. A randomized, doubleblind trial of $2.5 \mathrm{mg}$ and $5 \mathrm{mg}$ vortioxetine (Lu AA21004) versus placebo for 8 weeks in adults with major depressive disorder. Curr Med Res Opin. 2013;29:217-226.

54. Boulenger JP, Loft H, Olsen CK. Efficacy and safety of vortioxetine (Lu AA21004), 15 and 20 mg/day: a randomized, double-blind, placebocontrolled, duloxetine-referenced study in the acute treatment of adult patients with major depressive disorder. Int Clin Psychopharmacol. 2014;29:138-149.

55. McIntyre RS, Lophaven S, Olsen CK. A randomized, double-blind, placebo-controlled study of vortioxetine on cognitive function in depressed adults. Int J Neuropsychopharmacol. 2014;17:1557-1567. 
56. Montgomery SA, Nielsen RZ, Poulsen LH, Haggstrom L. A randomised, double-blind study in adults with major depressive disorder with an inadequate response to a single course of selective serotonin reuptake inhibitor or serotonin-noradrenaline reuptake inhibitor treatment switched to vortioxetine or agomelatine. Hum Psychopharmacol. 2014;29:470-482.

57. Baldwin DS, Loft H, Dragheim M. A randomised, double-blind, placebo controlled, duloxetine-referenced, fixed-dose study of three dosages of Lu AA21004 in acute treatment of major depressive disorder (MDD) Eur Neuropsychopharmacol. 2012;22:482-491.

58. Jacobsen PL, Mahableshwarkar AR, Serenko M, Chan S, Trivedi MH. A randomized, double-blind, placebo-controlled study of the efficacy and safety of vortioxetine $10 \mathrm{mg}$ and $20 \mathrm{mg}$ in adults with major depressive disorder. J Clin Psychiatry. 2015;76:575-582.

59. Mahableshwarkar AR, Jacobsen PL, Serenko M, Chen Y, Trivedi MH A randomized, double-blind, placebo-controlled study of the efficacy and safety of 2 doses of vortioxetine in adults with major depressive disorder. J Clin Psychiatry. 2015;76:583-591.

60. Montgomery SA, Asberg M. A new depression scale designed to be sensitive to change. Br J Psychiatry. 1979;134:382-389.

61. Hamilton M. A rating scale for depression. J Neurol Neurosurg Psychiatry. 1960;23:56-62.

62. Berhan A, Barker A. Vortioxetine in the treatment of adult patients with major depressive disorder: a meta-analysis of randomized double-blind controlled trials. BMC Psychiatry. 2014;14:276.

63. Pae CU, Wang SM, Han C, et al. Vortioxetine: a meta-analysis of 12 short-term, randomized, placebo-controlled clinical trials for the treatment of major depressive disorder. J Psychiatry Neurosci. 2015;40 174-186.

64. Citrome L. Vortioxetine for major depressive disorder: a systematic review of the efficacy and safety profile for this newly approved antidepressant - what is the number needed to treat, number needed to harm and likelihood to be helped or harmed? Int J Clin Pract. 2014;68:60-82.

65. Llorca PM, Lancon C, Brignone M, et al. Relative efficacy and tolerability of vortioxetine versus selected antidepressants by indirect comparisons of similar clinical studies. Curr Med Res Opin. 2014;30:2589-2606

66. Fu J, Chen Y. The efficacy and safety of $5 \mathrm{mg} / \mathrm{d}$ vortioxetine compared to placebo for major depressive disorder: a meta-analysis. Psychopharmacology (Berl). 2015;232:7-16.

67. Baldwin DS, Hansen T, Florea I. Vortioxetine (Lu AA21004) in the long-term open-label treatment of major depressive disorder. Curr Med Res Opin. 2012;28:1717-1724.

68. Alam MY, Jacobsen PL, Chen Y, Serenko M, Mahableshwarkar AR. Safety, tolerability, and efficacy of vortioxetine (Lu AA21004) in major depressive disorder: results of an open-label, flexible-dose, 52-week extension study. Int Clin Psychopharmacol. 2014;29 36-44.

69. Boulenger JP, Loft H, Florea I. A randomized clinical study of Lu AA21004 in the prevention of relapse in patients with major depressive disorder. J Psychopharmacol. 2012;26:1408-1416.

70. Rosenberg KP, Bleiberg KL, Koscis J, Gross C. A survey of sexual side effects among severely mentally ill patients taking psychotropic medications: impact on compliance. J Sex Marital Ther. 2003;29:289-296.

71. Williams VS, Baldwin DS, Hogue SL, Fehnel SE, Hollis KA, Edin HM. Estimating the prevalence and impact of antidepressant-induced sexual dysfunction in 2 European countries: a cross-sectional patient survey. J Clin Psychiatry. 2006;67:204-210.

72. van Geffen EC, van der Wal SW, van Hulten R, de Groot MC, Egberts AC, Heerdink ER. Evaluation of patients' experiences with antidepressants reported by means of a medicine reporting system. Eur J Clin Pharmacol. 2007;63:1193-1199.

73. McGahuey CA, Gelenberg AJ, Laukes CA, et al. The Arizona Sexual Experience Scale (ASEX): reliability and validity. J Sex Marital Ther. 2000;26:25-40.

74. Warner CH, Bobo W, Warner C, Reid S, Rachal J. Antidepressant discontinuation syndrome. Am Fam Physician. 2006;74:449-456.
75. Zajecka J, Tracy KA, Mitchell S. Discontinuation symptoms after treatment with serotonin reuptake inhibitors: a literature review. J Clin Psychiatry. 1997;58:291-297.

76. Black K, Shea C, Dursun S, Kutcher S. Selective serotonin reuptake inhibitor discontinuation syndrome: proposed diagnostic criteria. J Psychiatry Neurosci. 2000;25:255-261.

77. Rosenbaum JF, Fava M, Hoog SL, Ascroft RC, Krebs WB. Selective serotonin reuptake inhibitor discontinuation syndrome: a randomized clinical trial. Biol Psychiatry. 1998;44:77-87.

78. Jacobsen P, Mahableshwarkar A, Serenko M, Chan S, Trivedi M. Efficacy and safety of vortioxetine $10 \mathrm{mg}$ and $20 \mathrm{mg}$ in an 8-week, randomized, double-blind, placebo-controlled study of adults with major depressive disorder. Available from: http://www.psychcongress. $\mathrm{com} /$ posters/efficacy-and-safety-vortioxetine-10mg-and-20mg-8-weekrandomized-double-blind-placebo. Accessed July 22, 2015.

79. Mahableshwarkar A, Jacobsen P, Serenko M, Chen Y, and Trivedi M. Efficacy and safety of vortioxetine 15 and $20 \mathrm{mg}$ in an 8-week placebo-controlled and duloxetine-referenced trial of adults with major depressive disorder. Available from: http://www.psychcongress. com/posters/efficacy-and-safety-vortioxetine-15-and-20-mg-8-weekplacebo-controlled-and-duloxetine. Accessed July 22, 2015.

80. Artigas F, Nutt DJ, Shelton R. Mechanism of action of antidepressants. Psychopharmacol Bull. 2002;36 Suppl 2:123-132.

81. Dawson LA, Watson JM. Vilazodone: a 5-HT1A receptor agonist/ serotonin transporter inhibitor for the treatment of affective disorders. CNS Neurosci Ther. 2009;15:107-117.

82. Duru G, Fantino B. The clinical relevance of changes in the MontgomeryAsberg Depression Rating Scale using the minimum clinically important difference approach. Curr Med Res Opin. 2008;24:1329-1335.

83. Zimmerman M, Chelminski I, Posternak MA. Generalizability of antidepressant efficacy trials: differences between depressed psychiatric outpatients who would or would not qualify for an efficacy trial. Am J Psychiatry. 2005;162:1370-1372.

84. Cipriani A, Furukawa TA, Salanti G, et al. Comparative efficacy and acceptability of 12 new-generation antidepressants: a multipletreatments meta-analysis. Lancet. 2009;373:746-758.

85. Girardi P, Pompili M, Innamorati M, et al. Duloxetine in acute major depression: review of comparisons to placebo and standard antidepressants using dissimilar methods. Hum Psychopharmacol. 2009;24:177-190.

86. Zhang J, Mathis MV, Sellers JW, et al. The US Food and Drug Administration's perspective on the new antidepressant vortioxetine. J Clin Psychiatry. 2015;76:8-14.

87. McIntyre RS. When should you move beyond first-line therapy for depression? J Clin Psychiatry. 2010;71 Suppl 1:16-20.

88. Papakostas GI, Thase ME, Fava M, Nelson JC, Shelton RC. Are antidepressant drugs that combine serotonergic and noradrenergic mechanisms of action more effective than the selective serotonin reuptake inhibitors in treating major depressive disorder? A meta-analysis of studies of newer agents. Biol Psychiatry. 2007;62:1217-1227.

89. Berlim MT, Turecki G. What is the meaning of treatment resistant/ refractory major depression (TRD)? A systematic review of current randomized trials. Eur Neuropsychopharmacol. 2007;17: 696-707.

90. Alvarez E, Perez V, Artigas F. Pharmacology and clinical potential of vortioxetine in the treatment of major depressive disorder. Neuropsychiatr Dis Treat. 2014;10:1297-1307.

91. Baldwin DS, Nutt DJ. On assessing potential efficacy for vortioxetine in generalized anxiety disorder. Eur Neuropsychopharmacol. 2012;22: $841-843$.

92. Hasin DS, Goodwin RD, Stinson FS, Grant BF. Epidemiology of major depressive disorder: results from the National Epidemiologic Survey on Alcoholism and Related Conditions. Arch Gen Psychiatry. 2005;62:1097-1106.

93. Kessler RC, Gruber M, Hettema JM, Hwang I, Sampson N, Yonkers KA. Co-morbid major depression and generalized anxiety disorders in the National Comorbidity Survey follow-up. Psychol Med. 2008:38:365-374. 
94. Bidzan L, Mahableshwarkar AR, Jacobsen P, Yan M, Sheehan DV. Vortioxetine (Lu AA21004) in generalized anxiety disorder: results of an 8-week, multinational, randomized, double-blind, placebo-controlled clinical trial. Eur Neuropsychopharmacol. 2012;22:847-857.

95. Rothschild AJ, Mahableshwarkar AR, Jacobsen P, Yan M, Sheehan DV. Vortioxetine (Lu AA21004) $5 \mathrm{mg}$ in generalized anxiety disorder: results of an 8-week randomized, double-blind, placebo-controlled clinical trial in the United States. Eur Neuropsychopharmacol. 2012;22:858-866.
96. Mahableshwarkar AR, Jacobsen PL, Serenko M, Chen Y. A randomized, double-blind, fixed-dose study comparing the efficacy and tolerability of vortioxetine 2.5 and $10 \mathrm{mg}$ in acute treatment of adults with generalized anxiety disorder. Hum Psychopharmacol. 2014;29:64-72.

97. Theunissen EL, Street D, Hojer AM, Vermeeren A, van OA, Ramaekers JG. A randomized trial on the acute and steady-state effects of a new antidepressant, vortioxetine (Lu AA21004), on actual driving and cognition. Clin Pharmacol Ther. 2013;93:493-501.

\section{Publish your work in this journal}

Therapeutics and Clinical Risk Management is an international, peerreviewed journal of clinical therapeutics and risk management, focusing on concise rapid reporting of clinical studies in all therapeutic areas, outcomes, safety, and programs for the effective, safe, and sustained use of medicines. This journal is indexed on PubMed Central, CAS,
EMBase, Scopus and the Elsevier Bibliographic databases. The manuscript management system is completely online and includes a very quick and fair peer-review system, which is all easy to use. Visit http://www.dovepress.com/testimonials.php to read real quotes from published authors.

Submit your manuscript here: http://www.dovepress.com/therapeutics-and-clinical-risk-management-journal 